\title{
Traffic Flow Registraton for Unmanned Aerial Vehicle Detection
}

$$
\text { Gui-Yuan XIAO }{ }^{1,2, a} \text {, Rong-Yi DU }{ }^{3, b, *}
$$

\author{
${ }^{1}$ School of Transportation Engineering, TongJi University, Shanghai, China, 201804; \\ ${ }^{2}$ Guangxi Key Laboratory of geomechnics and Geotechnical Engineering, Guilin University of \\ Technology, Guilin, China, 541004
}

${ }^{3}$ Nanning Municipal Public Security Bureau Transportation Science Engineering Institute Nanning, China, 530000

axiaoguiyuangit@163.com, ${ }^{b}$ dry1024w@163.com

*Corresponding author

Keywords: Intelligent Transportation, Unmanned Aerial Vehicles, Time-Space Registration, Traffic Information Collection, Time Effectiveness.

\begin{abstract}
Registration of detection data is to ensure the time-space consistency and effective fusion of air-ground traffic detection data. In this paper, we aim to propose a space-time registration method for the vehicle detection data of unmanned aerial vehicle (UAV). Considering temporal and spatial distribution characteristics of UAV detection, we define time effectiveness and two evaluation indicators for UAV data registration, namely absolute and relative effective times. These two indicators are used to characterize the quality of UAV detection data. Based on time effectiveness of UAV detection, space and time registration methods are developed. Data verification is conducted by Matlab programming with the holographic vehicle trajectory data of I-80 highway. Results of the case study show that, registration largely improves the data accuracy of UAV traffic flow detection data from $68.4 \%$ to $88.8 \%$.
\end{abstract}

\section{Introduction}

Aerial detection technologies show great potential in the road traffic information acquisition applications[1]. Although satellites are initially considered for traffic surveillance purposes, their applications in transportation are limited not only by the transitory nature of satellite orbits which lead to be difficult to obtain the right imagery to address continuous problems such as traffic tracking, but also by the bad image quality caused by the cloud covers[2]. Besides, manned aircrafts are deemed "expensive for traffic surveillance studies and non-operational in severe weathers as well as potentially unsafe environments due to presence and safety concern of operators" [3]. As a low cost (fixed and marginal costs) and flexible 'eye-in-the-sky', unmanned aerial vehicle (UAV) equipped with video cameras, geo-positioning sensors and communication hardware to relay data to the ground is a good alternative to monitor traffic and collect detailed space/temporal data in real-time for both off-line planning and real time management applications, which doesn't involve risk of operators[4]. However, there are few literatures to study the time effectiveness of UAV traffic collection information and the methodology for the calculation of road traffic parameters based on UAV detection.

\section{UAV Traffic Detection Technology}

\section{UAV Video Detection Technology}

UAV video detection technology, also known as UAV aerial photography technology, refers to the use of high-performance UAV which is integrated with GPS positioning system, wireless communication systems and high-resolution video camera system to obtain image/video data and extract required information. 


\section{Scenarios of UAV Detection}

When UAV is flying in the direction of traffic flow, according to the time sequence of UAV and vehicles entering and leaving a detected road, there are nine scenarios as shown in Fig. 2, where the dashed line denotes UAV trajectory on the detected road and the solid lines denote vehicle trajectories. In Fig.2, width $\boldsymbol{L}_{u}$ is the detection distance of UAV with a certain flying height, the length $\Delta \boldsymbol{t}$ is the time interval between the leading end and the tail end of the UAV detection region entering or leaving detected road. ${ }^{\boldsymbol{t}_{u i}}$ is the time of the leading end of the UAV detection region entering detected

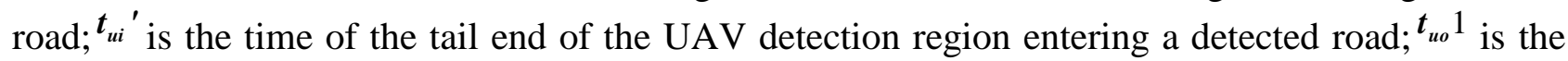
time of the leading end of the UAV detection region leaving detected road; ${ }^{{ }_{u o}}{ }^{2}$ is the time of the tail end of the UAV detection region leaving detected road; ${ }_{v i 1}, \boldsymbol{t}_{v i 2}$ and $\boldsymbol{t}_{v i 3}$ are the times of vehicles entering a detected road; ${ }^{\boldsymbol{t}_{v 01}}, \boldsymbol{t}_{{ }{ }^{2} 2}$ and $\boldsymbol{t}_{v 03}$ are the times of vehicles leaving detected road; and $\boldsymbol{L}_{\text {is }}$ the length of detected road.

\section{Time Effectiveness UAV Detection}

\section{Definition and Indicators of Time Effectiveness}

The analysis of Section II shows that, UAV traffic detection in flight is spatially distributed, i.e. UAV traffic detection system in flight state is based on mobile detection region for vehicle information sampling. So it will make the collected vehicle data distributed in time and space. The spatial and temporal distribution of detected vehicles and the detected vehicle trajectory (length) not only depends on UAV flight status (direction, height and speed, etc.), but also directly depends on traffic operation status on the detected road (such as vehicle speed and vehicle distribution).
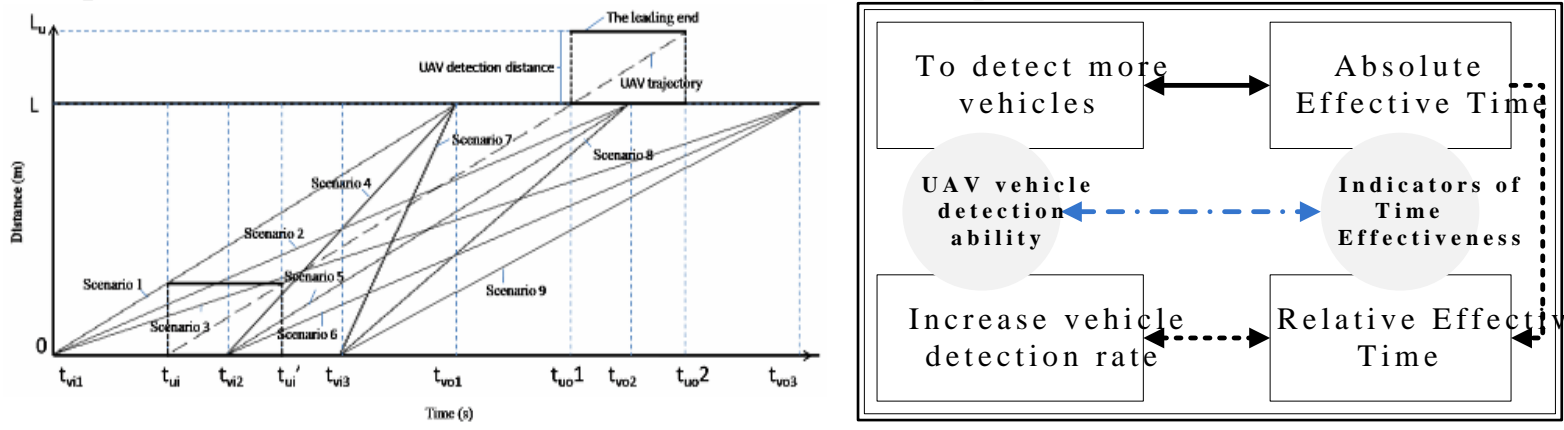

Fig.1 Time and space variation diagram of Fig.2 UAV time effectiveness indicators and UAV detection region their symbolical meanings

In order to characterize and investigate the impact of various UAV detection states and UAV flight parameter setting on road vehicle detection, the definition of time effectiveness was proposed. Effective time (ET) for UAV vehicle detection refers to the time span of the vehicles detected by UAV passing through some road section. Two indicators are introduced to evaluate the ET of UAV detection, i.e. absolute effective time length (AET) and relative effective time length (RET). AET is defined as the time interval of the vehicles detected by UAV passing through a detected road section, while RET is defined as the ratio of AET and the detection time of UAV on a detected road. The UAV test data to be effective and relatively effective time is used to measure the vehicle detection capability of UAV in various detection states. As shown in Fig. 2, AET of UAV vehicle detection data will be used to characterize the capability of detecting the number of vehicles, while RET will be used to characterize the UAV vehicle detection rate, which takes in account the time interval when vehicles on objective road can be detected and detection time of UAV.

\section{Effective Time Analysis of UAV Detection}

First, analyzing the ET of vehicle data based on UAV detection in same direction. Assuming $\boldsymbol{V}_{u s}, \boldsymbol{V}_{u f}$ and $\boldsymbol{V}_{u e}$ as the flight speeds of UAV, and there are $V_{u s}<V_{v}, V_{u f}>V_{v}$ and $V_{u e}=V_{v}$.

(1) When $V_{u}=V_{u s}<V_{v}$ 
As shown in Fig. 3, if the vehicles with speed $\boldsymbol{V}_{v}$ can be detected by UAV with flight speed $\boldsymbol{V}_{u s}$, a condition should be satisfied that,

$$
\left\{\begin{array}{l}
t_{v i}>t_{u i} \\
t_{v o}<t_{u o}
\end{array} .\right.
$$

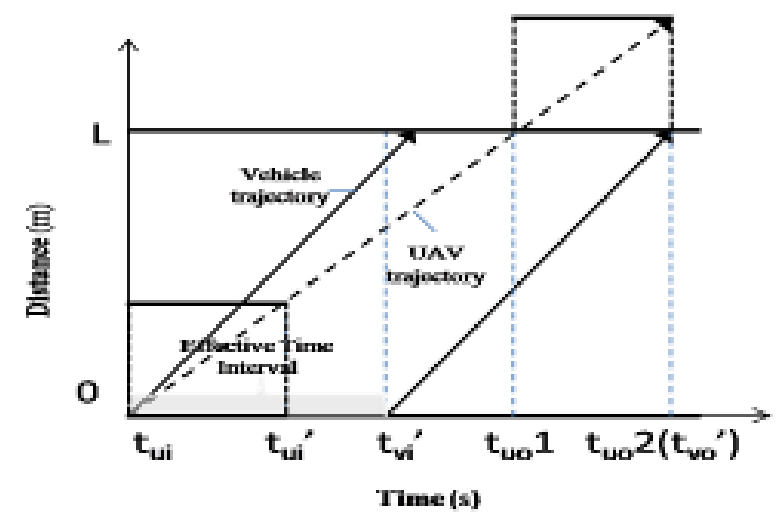

Fig.3 Time intervals entering detected road of detected vehicles in same direction

Obviously, when $t_{v o}=t_{u o} 2$, there are

$$
\left\{\begin{array}{l}
V_{v}=\frac{L}{t_{v o}{ }^{\prime}-t_{v i}{ }^{\prime}} \\
t_{v o}{ }^{\prime}=t_{u o} 2
\end{array} .\right.
$$

Then the time of the detected vehicles entering a detected road reaches the maximum $t_{v i}^{\max }$,

$$
t_{v i}^{\max }=t_{v i}{ }^{\prime}=\frac{V_{v} t_{u o} 2-L}{V_{v}}=t_{u o} 2-\frac{L}{V_{v}} .
$$

So, when the vehicle speed is greater than the UAV speed, the time interval of the detected vehicles entering a detected road is $\left(t_{u i}, \frac{V_{v} t_{u o} 2-L}{V_{v}}\right)$.

Let $\Delta t_{f}=t_{v i}^{\max }-t_{v i}^{\min }$ and there is,

$$
\Delta t_{f}=t_{u o} 2-\frac{L}{V_{v}}-t_{u i}=\frac{L}{V_{u s}}+\frac{L_{u}}{V_{u s}}-\frac{L}{V_{v}} .
$$

Hence, $\Delta t_{f}$ is the AET of the detected vehicles with the speed $v_{v}$, when the UAV flight speed is set smaller.

The corresponding RET is

$$
\lambda_{f}=\frac{\Delta t_{f}}{\Delta t_{u}}=\frac{\frac{L_{u}}{V_{u s}}+\frac{L}{V_{u s}}-\frac{L}{V_{v}}}{\frac{L+L_{u}}{V_{u s}}}=1-\frac{L}{L+L_{u}} \cdot \frac{V_{u s}}{V_{v}} .
$$

(2)When $\boldsymbol{V}_{u}=\boldsymbol{V}_{u f}>\boldsymbol{V}_{v}$

we can figure out that

$$
\Delta t_{s}=t_{u i}{ }^{\prime}-\left(t_{u o} 1-\frac{L}{V_{v}}\right)=\frac{L_{u}}{V_{u f}}-\frac{L}{V_{u f}}+\frac{L}{V_{v}} .
$$

Hence, $\Delta t_{s}$ is the AET of the detected vehicles with speed $v_{v}$, when setting a larger UAV flight speed.

Thus, the corresponding RET is 


$$
\lambda_{s}=\frac{\Delta t_{s}}{\Delta t_{u}}=\frac{\frac{\boldsymbol{L}_{u}}{V_{u f}}-\frac{\boldsymbol{L}}{V_{u f}}+\frac{\boldsymbol{L}}{\boldsymbol{V}_{v}}}{\frac{\boldsymbol{L}+\boldsymbol{L}_{u}}{\boldsymbol{V}_{u f}}}=\frac{\boldsymbol{L}_{u}-\boldsymbol{L}}{\boldsymbol{L}+\boldsymbol{L}_{u}}+\frac{\boldsymbol{L}}{\boldsymbol{L}+\boldsymbol{L}_{u}} \cdot \frac{\boldsymbol{V}_{u f}}{V_{v}} .
$$

(3) When $\boldsymbol{V}_{u}=\boldsymbol{V}_{u e}=\boldsymbol{V}_{v}$

So $\Delta t_{e}$ is the AET of the detected vehicles with the same speed as UAV. The corresponding RET is equal to

$$
\lambda_{e}=\frac{\Delta t_{e}}{\Delta t_{u}}=\frac{t_{u i}{ }^{\prime}-t_{u i}}{t_{u o} 2-t_{u i}}=\frac{L_{u}}{L+L_{u}} .
$$

Obviously, $\lambda_{e}<1$.

From the above analyses, we can summarize that the AET and the RET of UAV vehicle detection data in same direction are shown in below.

Applying the same method, we can find that when UAV detect traffic flow in reverse direction, the time interval of the detected vehicles with speed $\boldsymbol{V}_{v}$ entering a detected road is $\left(\boldsymbol{t}_{u i}-\frac{\boldsymbol{L}}{\boldsymbol{V}_{v}}, \boldsymbol{t}_{u \boldsymbol{v}} 2\right)$ and the corresponding AET $\Delta t_{r e v}$ and RET $\lambda_{r e v}$ are

$$
\begin{gathered}
\left\{\begin{array}{ccc}
A E T & R E T & \text { Cond } \\
\frac{L}{V_{u}}+\frac{L_{u}}{V_{u}}-\frac{L}{V_{v}} & 1-\frac{L}{L+L_{u}} \cdot \frac{V_{u}}{V_{v}} & V_{u}<V_{v} \\
\frac{L_{u}}{V_{u}}-\frac{L}{V_{u}}+\frac{L}{V_{v}} & \frac{L_{u}-L}{L+L_{u}}+\frac{L}{L+L_{u}} \cdot \frac{V_{u}}{V_{v}} & V_{u}>V_{v} \\
\frac{L_{u}}{V_{u}} & \frac{L_{u}}{L+L_{u}} & V_{u}=V_{v}
\end{array}\right. \\
\begin{cases}\Delta t_{r e v}=t_{u o} 2-\left(t_{u i}-\frac{L}{V_{v}}\right)=\frac{L}{V_{u}}+\frac{L_{u}}{V_{u}}+\frac{L}{V_{v}} \\
\lambda_{r e v}=\frac{\Delta t_{r e v}}{\Delta t_{u}}=\frac{\frac{L_{u}}{V_{u}}+\frac{L}{V_{u}}+\frac{L}{V_{v}}}{\frac{L+L_{u}}{V_{u}}}=1+\frac{L}{L+L_{u}} \cdot \frac{V_{u}}{V_{v}}\end{cases}
\end{gathered}
$$

Obviously, $\lambda_{\text {rev }}>1$. The reason is that the detected vehicles can be divided into two parts: the vehicles which are already presented in detected road before UAV entering and the vehicles entering detected road within the UAV detection time.

\section{Traffic Flowdata Registration Method}

In Section III, time effectiveness of vehicle data from UAV detection was analyzed considering temporal and spatial distribution characteristics of vehicle detections. In order to estimate the road traffic parameters based on the obtained vehicle data from UAV detection and realize the fusion of air-ground traffic detection data, it is necessary to carry out space/time registration for UAV vehicle data based on the time effectiveness of UAV detection data to ensure the space-time correlation consistency of the detection data from UAV system and other sources.

\section{Space Registration}

Space registration of multi-source traffic data refers to the transformation of multi-source traffic data to a uniform spatial reference system, i.e. making the transformed traffic data associated with corresponding detection object (e.g., road section). After space registration, detection data from various sources would associate with same detection object. In this section, taking the space registration of traffic flow data between UAV detection and ground point detection for example to 
introduce the space registration oriented traffic data fusion of air-ground traffic detections. Usually, road traffic flow detection is based on ground fixed detectors, so the reference cross-section is set where there are fixed detectors. Hence, the space registration of traffic flow data between UAV detection and ground point detection mainly refers to the transformation of UAV detection data into traffic flow data of the cross-section where is ground fixed detector.

Supposing the detected vehicle number by UAV detection is $N$ and the driving distance, first time and last time of the $\boldsymbol{k} \boldsymbol{t} \boldsymbol{h}(\boldsymbol{k}=1,2,3, \ldots, N)$ vehicle detected by UAV on objective road are $\boldsymbol{d}_{\boldsymbol{k}}, \boldsymbol{t}_{1 \boldsymbol{k}}$ and $t_{2 k}$ respectively, the driving time $t_{k}$ of the $k t h$ vehicle is equal to

$$
t_{k}=t_{2 k}-t_{1 k} .
$$

Applying the calculation approach proposed by Edie [5], the average speed of the road traffic flow

$$
V_{v}=\sum_{k=1}^{N} d_{k} / \sum_{k=1}^{N} t_{k}
$$

Supposing the tịme when UAV entering the objective road with the length $\boldsymbol{L}$ is $\boldsymbol{t}_{u i}$, the detection distance is $\boldsymbol{L}_{u}$ and the flight speed is $\boldsymbol{V}_{\boldsymbol{u}}$, we can know the time of the leading end of the UAV detection region leaving detected road $t_{u o} 1$ is equal to

$$
\boldsymbol{t}_{u o} 1=\frac{\boldsymbol{L}}{V_{u}}+\boldsymbol{t}_{u i}
$$

And the time of the tail end of the UAV detection region leaving detected road $t_{u o} 2$ is

$$
t_{u o} 2=\frac{L+L_{u}}{V_{u}}+t_{u i}=t_{u o} 1+\frac{L_{u}}{V_{u}} .
$$

Meanwhile, based on Formula (1), we can figure out the time of the tail end of the UAV detection region entering the objective road, which is shown in Formula (13).

$$
\boldsymbol{t}_{u i}{ }^{\prime}=\boldsymbol{L}_{u} / \boldsymbol{V}_{u}+\boldsymbol{t}_{u i} \text {. }
$$

So, based on the conclusion of Section 3.2, the transformation of UAV vehicle data into the traffic flow information of a cross-section where there are ground fixed detectors will be carried out under the following rules:

i. when UAV flight direction is the same with traffic flow:

(1) if $\boldsymbol{V}_{v}>\boldsymbol{V}_{u}$ : the detected vehicles by UAV is corresponding to the vehicles entering the objective cross-section within the time interval $\left(t_{u i},\left(V_{v} t_{u o} 2-L\right) / V_{v}\right)$;

(2) if $\boldsymbol{V}_{v} \leq \boldsymbol{V}_{u}$ : the detected vehicles by UAV is corresponding to the vehicles entering the objective cross-section within the time interval $\left(\left(\boldsymbol{V}_{v} \boldsymbol{t}_{u o} 1-\boldsymbol{L}\right) / \boldsymbol{V}_{v}, \boldsymbol{t}_{u i}{ }^{\prime}\right)$.

ii. when UAV flight direction is opposite to traffic flow, the detected vehicles by UAV is corresponding to the vehicles entering the objective cross-section within the time interval $\left(t_{u i}-\frac{L}{V_{v}}, t_{u o} 2\right)$.

Obviously, space registration of UAV traffic flow data is mainly to identify effective time interval which is corresponding to the time when the detected vehicles driving through an objective cross-section. The obtained effective time interval of UAV traffic flow data after space registration is generally not uniform with the sampling period or sampling time interval of ground fixed detector traffic flow data, so time registration is necessary to ensure the uniform of the aerial and ground detection data for fusion purpose or other application. 


\section{Time Registration}

Time registration of multi-source traffic data refers to the transformation of multi-source traffic data after space registration into a uniform time section division through the procession of accumulation and translation ${ }^{[6]}$.

Taking two detectors $\mathrm{A}$ and $\mathrm{B}$ as example, supposing their sampling periods are $\tau_{1}$ and $\tau_{2}$ respectively,

(1) If $\tau_{1}: \tau_{2}=\boldsymbol{n}$ ( $\boldsymbol{n}$ is positive integer)

As shown in Fig. 4, obviously, in the time interval between two consecutive sampling of detector A, detector B has $\boldsymbol{n}$ times of sampling, that is, detector B has $\boldsymbol{n}$ sampling data.

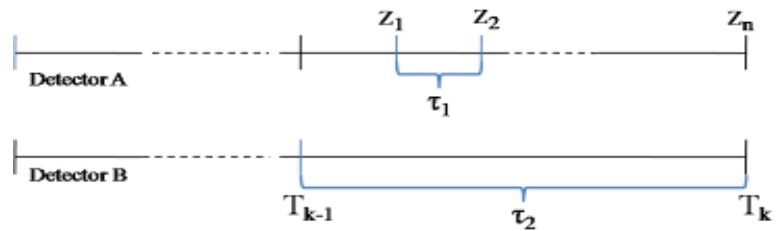

Fig.4 Sampling period of integer times contrast diagram

There are two methods to carry out time registration for the detection data of detectors A and B: adding method and least square method ${ }^{[7]}$. According to adding method, the virtual measurement values of detector B will be taken as the sums of its $\boldsymbol{n}$ sampling data in each sampling period of detector A. As for least-squares method, the virtual measurement values of detector B will be the results of the $\boldsymbol{n}$ sampling data in each sampling period of detector A based on least square rule. Both methods are simple and practical, but not suitable for the situation that the data of detectors for time registration have different time base and don't conform to the requirement of integer times of sampling periods.

(1)In general case

the data of detectors for time registration have different time base and don't conform to the requirement of integer times of sampling periods. .For this case, Wang et al. ${ }^{[8]}$ proposed an interpolation and extrapolation method for the time registration of multiple target tracking data. This method is to form a series of equally spaced targets observation data through interpolation and extrapolation operation of high accuracy observation data in each time slice. The time registration method is shown in formula (15).

$$
\left\{\begin{array}{l}
\boldsymbol{x}_{\tau_{i} t_{j}}=\boldsymbol{x}_{\tau_{i}}+\left(\boldsymbol{t}_{j}-\tau_{i}\right) \boldsymbol{v}_{x \tau_{i}} \\
\boldsymbol{y}_{\tau_{i} t_{j}}=\boldsymbol{y}_{\tau_{i}}+\left(\boldsymbol{t}_{j}-\tau_{i}\right) \boldsymbol{v}_{\boldsymbol{y} \tau_{i}} \\
\boldsymbol{z}_{\tau_{\tau_{i} t_{j}}}=\boldsymbol{z}_{\tau_{\tau_{i}}}+\left(\boldsymbol{t}_{j}-\tau_{i}\right) \boldsymbol{v}_{z \tau_{i}}
\end{array} .\right.
$$

Where, $\left(\boldsymbol{x}_{\tau_{1}}, \boldsymbol{y}_{\tau_{1}}, z_{\tau_{1}}, \boldsymbol{v}_{x \tau_{1}}, \boldsymbol{v}_{y \tau_{1}}, \boldsymbol{v}_{z \tau_{1}}\right)$ is the target observation data of detector A, $\boldsymbol{x}_{\tau_{i} t_{j}}, \boldsymbol{y}_{\tau_{i} t_{j}}$ and $z_{\tau_{i} t_{j}}$ is $\boldsymbol{x}_{\tau_{1}}, \boldsymbol{y}_{\tau_{1}}, z_{\tau_{1}}, i \in[1, \boldsymbol{m}], \boldsymbol{j} \in[1, \boldsymbol{n}]$.

Extend the interpolation and extrapolation method to the time registration of traffic flow data of UAV and ground fixed detectors. Corresponding to some sampling time $\boldsymbol{k}$, the number of the detected vehicles by UAV is $\boldsymbol{x}_{\boldsymbol{k}}$ and the effective time interval is $\left[\boldsymbol{t}_{k}, \boldsymbol{t}_{k}+\tau_{k}\right] . \tau_{k}$ is the absolute effective time of the UAV vehicle data. So the value of the UAV data in sampling time $\boldsymbol{k}$ after registration can be figured out according to Formula (15).

$\overline{\boldsymbol{x}}_{k}=\boldsymbol{x}_{\boldsymbol{k}}+\left(\boldsymbol{T}-\tau_{k}\right) \boldsymbol{v}_{k}$.

Where, $\bar{x}_{k}$ is $\boldsymbol{x}_{\boldsymbol{k}}$ after time registration; $\boldsymbol{T}$ is the sampling period of ground fixed detector; $\boldsymbol{v}_{\boldsymbol{k}}$ is the vehicle detection rate of $\mathrm{UAV}, v_{k}=x_{k} / \tau_{k}$. 


\section{Data Verification}

The data developed by Cambridge Systematics, Inc. as part of the Federal Highway Administration's (FHWA) Next Generation Simulation (NGSIM) project [9]were used for data verification. The data include detail vehicle trajectory data and are collected by seven video cameras mounted on a 30-storey building, Pacific Park Plaza, which is located in 6363 Christie Avenue and adjacent to the Interstate freeway I-80. The sampling interval is 0.1 seconds and the data properties include vehicle ID, vehicle length, time, geographical coordinates, lane number and so on so forth. In addition, the road length $\boldsymbol{L}$ is about $535.7 \mathrm{~m}$ and the average speed of the vehicles on the detected road is $6 \mathrm{~m} / \mathrm{s} \sim 9 \mathrm{~m} / \mathrm{s}$. We set that UAV flew in the same direction with traffic flow, the detection distance $\boldsymbol{L}_{\boldsymbol{u}}$ is $88.5 \mathrm{~m}$ and the flight speed $\boldsymbol{V}_{\boldsymbol{u}}$ is $5 \mathrm{~m} / \mathrm{s}$. Meanwhile, to facilitate the calculation and verification, the sampling period of section flow detection is set as $125 \mathrm{~s}$ which is equal to $\Delta t_{u}$. Through Matlab programming simulation, comparing with the real traffic flow data, the average detection rate of UAV is 68.4 percent, i.e. the detection error is 31.6 percent. Based on the vehicle trajectory data obtained by UAV detection, link average speeds can be estimated using Formula (21) and the results are shown in Fig. 5. Obviously, $\boldsymbol{V}_{v}>\boldsymbol{V}_{u}$. Based on the results of Section 3.2, the estimated AET values of the UAV detection data can be obtained using Formula (17)

$$
\Delta t_{f} \approx \frac{L}{V_{u}}+\frac{L_{u}}{V_{u}}-\frac{L}{V_{v}} .
$$

Let $\boldsymbol{T}=\Delta \boldsymbol{t}_{u}, \tau_{k}=\Delta \boldsymbol{t}_{f}$, Formula (16) can be changed into Formula (18).

$$
\overline{\boldsymbol{x}}_{k}=\left(1+\frac{\boldsymbol{L}}{\left(\frac{\boldsymbol{L}}{\boldsymbol{V}_{u}}+\frac{\boldsymbol{L}_{u}}{\boldsymbol{V}_{u}}-\frac{\boldsymbol{L}}{\overline{\boldsymbol{V}}_{v s}}\right) \overline{\boldsymbol{V}}_{v s}}\right) \boldsymbol{x}_{k} .
$$

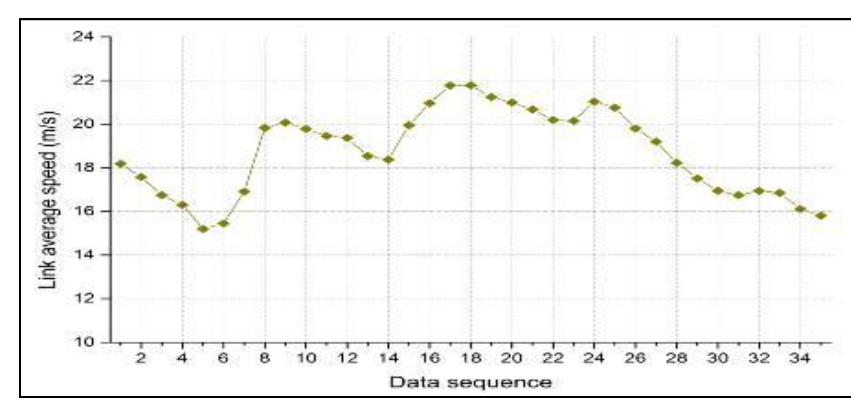

Fig. 5 Estimated link average speeds based on UAV detection

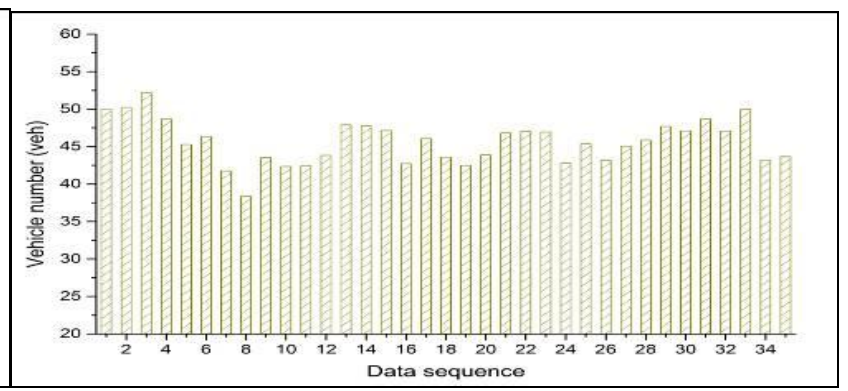

Fig.6 Traffic flow data of UAV detection after registration

The vehicle data of UAV detection after registration are shown in Fig. 6.

After registration, the average error of traffic flow data by UAV detection is 11.2 percent, which shows great improvement of the traffic flow precision for UAV detection.

\section{Conclusions}

In this paper, we have analyzed the UAV detection characteristic and proposed two time effectiveness evaluation indicators for UAV vehicle detection. Through vehicle detection scene analysis and theoretical derivation, we investigate the impacts of UAV flying parameter settings (e.g. detection distance and speed), road length, and vehicle speed on time effectiveness of UAV vehicle detection data. Subsequently, based on the time effectiveness analysis of UAV detection, space and time registration methods are developed. Finally, by Matlab programming with the holographic vehicle trajectory data of I-80, we carry out a registration example of UAV detection data. In the case study, it was shown that, the registration enhances the data accuracy of UAV traffic flow detection data from $68.4 \%$ to $88.8 \%$. The future research can be envisaged as follows. First, although the 
registration result is promising, we still can improve the effectiveness and applicability of the proposed registration model by considering the characteristics of traffic flow. Second, to address a practical traffic scenario, real UAV data registration study should be conducted and compared with the simulation results in this paper.

\section{References}

[1] Z.S. Yang. Basic traffic information fusion technology and its applications. Beijin: China Railway Publishing House, 2005.

[2] Gerhardinger, A., Ehrlich, D., and M. Pesaresi. Vehicles detection from very high resolution satellite imagery. In: International Archives of Photogrammetry and Remote Sensing, Vol. XXXVI, No. E/W24, 2005, pp. 83-88.

[3] Srinivasan, S., Latchman, H., Shea, J., et al. Airborne traffic surveillance systems: video surveillance of highway traffic. In proceedings of the ACM 2nd international workshop on video surveillance \& sensor networks, 2004, pp. 131-135.

[4] Coifman, B., McCord, M., Mishalani, R.G., and K. Redmill. Surface transportation surveillance from unmanned aerial vehicles. Presented at 83rd Annual Meeting of Transportation Research Board, Washington, D. C., 2004.

[5] L. Edie. Discussion of traffic stream measurements and definitions. In proceedings of second international symposium on the theory of traffic flow, OECD, 1963, pp. 139-154.

[6] Luo, R.C., and M.G. Kay. Multisensor integration and fusion in intelligent systems. IEEE transactions on systems, man, and cybernetics, Vol. 19, No. 5, 1989, pp. 901-931.

[7] Wang, J.J., and J.J. Huang. A spatial alignment algorithm for time varying biases of radar and infrared sensors. Procedia engineering, Vol. 15, 2011, pp. 2614-2618.

[8] Wang, B.S., and F.S. Li. Multiple target tracking algorithm based on data fusion technology (in Chinese). Journal of Xidian University, Vol. 25, No. 3, 1998, pp. 269-272.

[9] Federal Highway Administration. Traffic analysis tools: Next generation simulation (NGSIM). Available at: http://ops.fhwa.dot.gov/trafficanalysistools/ngsim.htm, accessed on May 16, 2013. 